\title{
Correlation of C-X-C chemokine receptor 2 upregulation with poor prognosis and recurrence in human glioma
}

This article was published in the following Dove Press journal:

OncoTargets and Therapy

2 November 2015

Number of times this article has been viewed

\author{
Liu Yang' \\ Zenghui Liu' \\ Ronghua $\mathrm{Wu}^{2}$ \\ Qi Yao' \\ Zhikai Gu' \\ Mei Liu ${ }^{2}$ \\ 'Department of Neurosurgery, \\ Affiliated Hospital of Nantong \\ University, ${ }^{2}$ Jiangsu Key Laboratory \\ of Neuroregeneration, Co-innovation \\ Center of Neuroregeneration, \\ Nantong University, Nantong, Jiangsu, \\ People's Republic of China
}

Correspondence: Mei Liu Jiangsu Key Laboratory of Neuroregeneration, Co-innovation Center of Neuroregeneration, Nantong University, No 19 Qixiu Road, Nantong, Jiangsu 22600I, People's Republic of China

Tel +8651385051852

Fax +8651385511585

Email liumei@ntu.edu.cn

\begin{abstract}
C}-\mathrm{X}-\mathrm{C}$ chemokine receptor 2 (CXCR2), a member of the G-protein-coupled receptor family, is an interleukin- 8 receptor and results in the activation of neutrophils. To date, CXCR2 has been identified with many cell events, including inflammation, neovascularization, metastasis, and cell carcinogenesis. This study aimed to investigate alterations in the expression of CXCR2 in patients with brain gliomas and relationships with pathological grades and clinicopathological characteristics. Brain tissue specimens from 60 patients with glioma and 15 patients undergoing surgery for epilepsy (controls) were detected using streptavidin-perosidase immunohistochemistry. Western blotting was used to evaluate CXCR2 protein levels with fresh tissues derived from glioma cases or controls. Correlations between CXCR2 expression and clinicopathological characteristics were analyzed using SPSS software. The results showed high-grade gliomas with high CXCR2 expression as compared with normal tissues. The expression of CXCR2 was significantly related to high grades and recurrence of tumor but not to age or sex. During an in vitro wound healing assay, U251 migration was reduced when the CXCR2-specific inhibitor SB225002 was applied. Our results suggested that the high expression of CXCR2 in gliomas was closely correlated to the degree of malignancy and recurrence and that CXCR2 inhibition decreased the migration of glioma cells. Therefore, CXCR2 may serve as a potential therapeutic target for the recurrence and migration of gliomas.
\end{abstract}

Keywords: CXCR2, human glioma, recurrence, cell migration

\section{Introduction}

Cerebral gliomas are known to be the most common tumors of the central nervous system, accounting for up to $50 \%$ of all intracranial tumors. ${ }^{1}$ Gliomas are clinically characterized by a high incident rate, a high recurrence rate, and high mortality., ${ }^{2,3}$ Complete surgical resection of these tumors is difficult, and postoperative relapses are frequent since they invade adjacent normal brain tissue that results in no obvious boundary between the normal tissue and the glioma. Treatment efficacy is still unsatisfactory, despite improvements in conventional treatments (eg, surgery, radiotherapy, and chemotherapy) and the emergence of new biological therapies (eg, gene therapy and immune therapy). ${ }^{3-6}$ Recently, relationships among the biological behaviors of gliomas, such as high proliferation, invasion, and immune suppression, have been the focus of much research. The invasiveness of malignant gliomas greatly contributes to the challenges involved in its treatment.

C-X-C chemokine receptor 2 (CXCR2), an interleukin-8 (IL8) receptor, possesses tumorigenic and proangiogenic properties that are similar to CXCR1, ${ }^{7}$ and it mediates neutrophil migration to sites of inflammation. ${ }^{8}$ In a previous study, glioblastoma cells secreted IL8 and were reported to induce permeability mediated through its 
receptor CXCR2 on brain endothelia. ${ }^{9}$ Raychaudhuri and Vogelbaum, ${ }^{10}$ using fluorescence-activated cell sorting analysis, reported that some brain tumor cell lines only expressed CXCR1 and not CXCR2. However, CXCR2 has been recently proven to be involved in the proliferation, migration, invasion, or metastasis of many malignant neoplasms, including melanomas and pancreatic, colorectal, lung, ovarian, prostate, brain, and renal cells. ${ }^{11-13}$ High expression levels of CXCR2 have been found in many of the tissue malignancies mentioned earlier. ${ }^{14-16}$ Korkolopoulou et $\mathrm{al}^{17}$ found that the presence of CXCR2 in astrocytoma adversely affected the survival of patients. It was found that mammary tumors and ovarian cancer underwent reduced growth, migration, or metastasis when using CXCR2-specific antagonists or with knockdown of CXCR2 expression. ${ }^{18,19}$ We know tumor recurrence still occurs in a high proportion of patients with glioblastoma. One reason might be attributable to the presence of cancer stem cells. Infanger et $\mathrm{al}^{20}$ used CXCR2 silencing in cancer stem cells from glioblastoma multiforme (GBM) and confirmed a critical role for the IL8/CXCR2 pathway in GBM pathogenesis through the promotion of malignant properties. Furthermore, Zhou et $\mathrm{al}^{21}$ found that although either CXCR1 or CXCR2 inhibition reduces tumor sphere (glioblastoma stem cell) formation, the IL8/CXCR1/ STAT3 pathway, but not CXCR2, is pivotal for stem-cell trait maintenance.

Based on the earlier studies, we considered that an independent clinical investigation of CXCR2 expression with different grades of glioma would be helpful for understanding the significance of CXCR2 expression during glioma progression. In this study, we reviewed and statistically analyzed the clinical characteristics of CXCR2 expression between the brain tissue of patients with glioma and nonglioma controls. Special attention was paid to the correlation between CXCR2 expression levels and the recurrence of gliomas. Moreover, we also observed the effect of the CXCR2-specific inhibitor (SB225002) on the migration of glioma cells in vitro to determine whether there was a response of CXCR2 inhibition on U251 cells.

\section{Materials and methods Ethics statement and patients with glioma}

The study was approved by the Clinical Research Ethics Committee of Nantong University Medical College. Sixty patients with glioma and 15 normal controls aged between 18 years and 76 years were recruited between February 2008 and September 2012 at the Department of Neurosurgery,
Affiliated Hospital of Nantong University, People's Republic of China. All patients underwent surgical tumor resection, and a diagnosis of glioma was confirmed by histological examination. Medical records and pathology reports of the 60 cases involving surgical resection of gliomas were reviewed and verified by the hospital senior pathologists. The cases were classified by clinical stage and histological type according to the 2007 World Health Organization classification of brain tumors. There were 29 cases with grades I-II tumors and 31 cases with grades III-IV tumors. Fifteen normal (control) brain tissues were also taken from patients admitted to our hospital in the same period with epilepsy or head injuries and with decompression lines. The Institutional Review Board of the author's establishment approved the protocol, and written informed consent was obtained from all patients before enrollment. The clinical records were reviewed, and information regarding age, sex, and recurrence was collected.

\section{Immunohistochemistry and interpretation of immunostaining results}

Formalin-fixed, paraffin-embedded sections of tissue specimens were reviewed by in-house neuropathologists. All slides were processed according to the manual from the HISTOSTAIN ${ }^{\circledR}$-SP kits (Thermo Fisher Scientific, Waltham, MA, USA). Briefly, serial 5- $\mu$ m sections were mounted on glass slides coated with $10 \%$ polylysine. The sections were deparaffinized with xylene and rehydrated in a graded series of ethanol. Endogenous peroxidase activity was blocked by soaking in 3\% hydrogen peroxide/methanol for 10 minutes. For antigen retrieval, the sections were processed in $10 \mathrm{mmol} / \mathrm{L}$ citrate buffer ( $\mathrm{pH} \mathrm{6.0)}$ ) and heated to $95^{\circ} \mathrm{C}$ in a microwave oven for 20 minutes. Subsequently, 10\% goat serum was applied for 1 hour of blocking, and the sections were then incubated overnight at $4^{\circ} \mathrm{C}$ with anti-human CXCR2 monoclonal antibody (diluted 1:100; Abcam, Cambridge, MA, USA). Phosphate-buffered saline (PBS) replaced the primary antibody as the negative control. The next day, the slides were rinsed in PBS, and biotinylated second antibodies, enzyme conjugate solution, and 3-amino-9-ethylcarbazole chromogen solution were used in sequence, respectively. After the specified time, the sections were counterstained with hematoxylin, dehydrated, and coverslipped. For evaluation of CXCR2 expression in different grades of tumors, ten randomly selected visual fields per section were examined at $\times 400$ magnification using a Leica light microscope (Leica Microsystems, Wetzlar, Germany). Five high-power fields in each specimen were randomly selected, and nuclear staining 
was examined under high-power magnification. More than 500 cells were counted to determine the mean percentage, which represented the percentage of immunostained cells relative to the total number of cells.

Immunohistochemical results were reviewed independently by two senior pathologists who were blinded as to the outcome of the tumors. Semiquantitative assessment of target proteins was performed by consensus and comprised both intensity of staining (negative as 0 , light yellow as 1 , brown as 2, tan as 3) for each cell and extent of staining (ratio of positive cells/counted cells, 1 for $<25 \%$, 2 for $25 \%-50 \%, 3$ for $51 \%-75 \%$, and 4 for $>75 \%$ ) for each random field. The scores for the intensity and extent of staining were multiplied to give a weighted score for each case (maximum possible, 12). For statistical analysis, the weighted scores were grouped into four categories where a score of 0 was considered negative, 1-4 (+) were regarded as low positive expression, and 5-8 (++), 9-12 (+++), (++)-(+++) were regarded as high positive expression.

\section{Protein extraction and Western blot analysis}

From the Affiliated Hospital of Nantong University, we collected four fresh brain tissue contusion samples and six fresh glioma tissue samples. Harvested tissues were stored in liquid nitrogen until their use in Western blot analysis. These cases were reevaluated for grade and histological type by the same two pathologists mentioned earlier.

Western blotting was performed according to standard protocols as described in our previous study. ${ }^{22}$ Tissues were cut into small pieces and homogenized in a glass homogenizer in extraction buffer provided in the protein extraction kit (Bio-Rad Laboratories Inc., Hercules, CA, USA) including $1 \%$ protease inhibitors (Bio-Rad Laboratories Inc.) and lysed on ice. After centrifugation, the protein concentration of the supernatant was analyzed using a bicinchoninic acid assay kit (Bio-Rad Laboratories Inc.). Forty micrograms of total protein from each sample was separated by sodium dodecyl sulfate-polyacrylamide gel electrophoresis and transferred to a polyvinylidene difluoride membrane (EMD Millipore, Billerica, MA, USA). Western blotting was performed according to standard protocols using the following antibodies: goat anti-glyceraldehyde 3-phosphate dehydrogenase polyclonal antibody (1:800; Santa Cruz Biotechnology Inc., Dallas, TX, USA), anti-human CXCR2 monoclonal antibody (1:500); donkey anti-goat IRDye (1:10,000; Rockland, Limerick, PA, USA), and donkey anti-mouse IRDye (1:10,000; Rockland). Immunoblots were analyzed using the Odyssey densitometry program (LI-COR, Lincoln, NE, USA). Glyceraldehyde 3-phosphate dehydrogenase was used as a loading control.

\section{CXCR2 inhibitor treatment and wound healing assay}

Human glioma cell lines (U251) were purchased from the cell bank at the Chinese Academy of Sciences (Shanghai, People's Republic of China). The U251 cells were cultured in Dulbecco's Modified Eagle's Medium (Thermo Fisher Scientific) with $10 \%$ fetal bovine serum, $2 \mathrm{mM}$ L-glutamine, and $1 \times$ penicillin-streptomycin (Thermo Fisher Scientific) in an incubator at $37^{\circ} \mathrm{C}$ and $5 \% \mathrm{CO}_{2}$. The cells were dissociated by a standard trypsinization procedure $(0.25 \%$ Trypsin + $0.02 \%$ ethylenediaminetetraacetic acid) and replated into 24-well plates. When the cells attached, a wound healing assay was performed. After washing with PBS, SB225002 (a CXCR2 inhibitor; final concentration $400^{\circ} \mathrm{nM}$ ) or an equal volume of dimethyl sulfoxide (served as control) was added. Then, at the indicated time points of 0 hour, 12 hours, 24 hours, 36 hours, and 48 hours, cells were observed and photographs were taken. The distance of the wound zone (cell-free void zone) was measured using ImageJ software as described in a previous study. ${ }^{23}$

\section{Statistical analyses}

All statistical analyses were performed using SPSS 19.0 software (IBM Corporation, Armonk, NY, USA). The Fisher's exact test was used to determine the significance between CXCR2 expression and clinicopathological parameters. Comparisons between with and without SB225002 treatment groups were analyzed by using repeated measures analysis of variance with a GraphPad Prism 5.0 software (GraphPad Software, Inc., La Jolla, CA, USA). The data were expressed as means \pm standard deviation and analyzed using one-way analysis of variance. The cut-off value for statistical significance was set at $P<0.05$.

\section{Results \\ High levels of CXCR2 protein expression were present in high-grade gliomas}

Immunohistochemistry of CXCR2 protein expression was performed in glioma specimens from 60 patients and nonglioma brain tissues from 15 control cases. As shown in Figure 1A, CXCR2-specific staining in cells was mostly found in the cytoplasm. CXCR2 expression appeared to be upregulated in gliomas. As shown in Figure $1 \mathrm{~B}$ and Table 1, the positive expression of CXCR2 protein was 

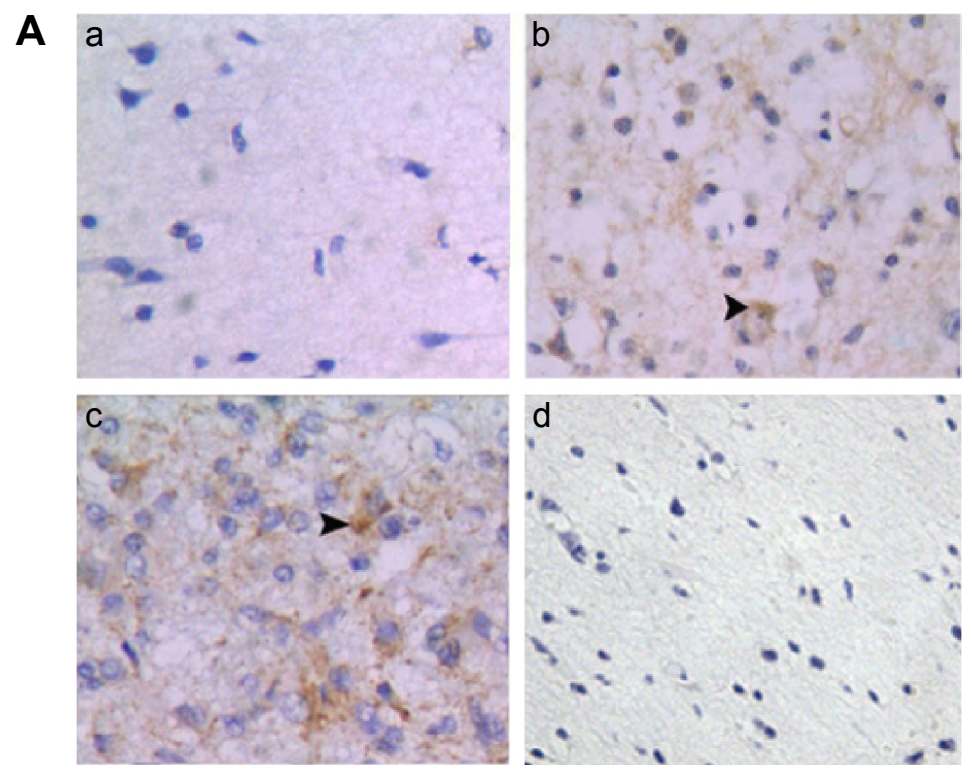

B

\section{Expression of CXCR2 in normal brain and different grades of gliomas}
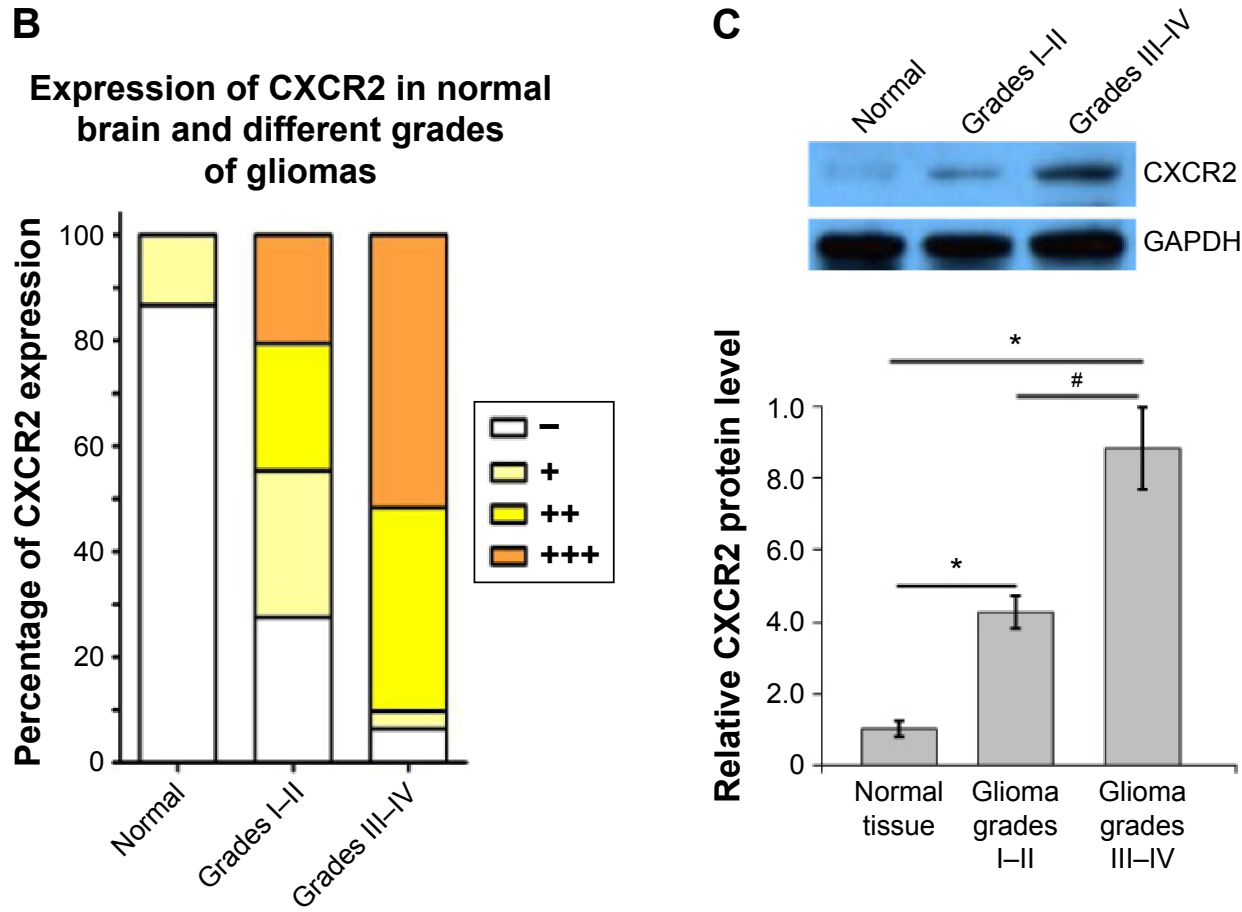

Figure I High level of CXCR2 expression shown in cases of high-grade gliomas.

Notes: (A) Representative sections for CXCR2 immunohistochemistry (IHC, SP $\times 400$ ). (a) Normal nontumorous tissue, (b) WHO low-grade glioma, (c) WHO high-grade glioma, (d) negative control for immunostaining. Positive CXCR2 staining = brown; cell nuclei = blue; the arrows show representative results of staining. (B) The population of cells with different levels of CXCR2 expression in glioma and control brain tissue. Overall, the level of CXCR2 expression was significantly higher in WHO III-IV gliomas tissues than in WHO I-II glioma tissues and the control brain tissues according to IHC results. (C) Western blotting of CXCR2 protein level in gliomas and normal tissue. The upper panel is a representative result of Western blotting. CXCR2 protein expression was calculated by normalizing CXCR2 intensity to GAPDH intensity, and data

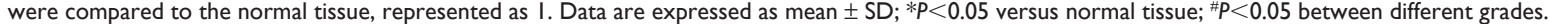

Abbreviations: CXCR2, C-X-C chemokine receptor 2; WHO, World Health Organization; GAPDH, glyceraldehyde 3-phosphate dehydrogenase; SD, standard deviation.

present in only $13.3 \%$ (two of 15 cases) in nonglioma control brain tissues, while present in $83.3 \%$ (50 of 60 cases) of glioma specimens. In glioma tissues, high levels of CXCR2 protein were expressed in high-grade tumors. Of the 60 cases of glioma, $90.3 \%$ ( 28 in 31 cases) of highgrade (III-IV) glioma tissues and $44.8 \%$ (13 in 29 cases) of low-grade (I-II) glioma tissues exhibited high CXCR2 expression. The expression of CXCR2 proteins in gliomas was significantly higher than that of nonglioma controls, and the expression of CXCR2 protein levels between grades I-II and grades III-IV gliomas was significantly different $(P<0.001)$. 
Table I Correlation of CXCR2 expression with clinicopathological characteristics in patients with glioma

\begin{tabular}{|c|c|c|c|c|}
\hline \multirow{2}{*}{$\begin{array}{l}\text { Clinicopathological } \\
\text { parameters }\end{array}$} & \multirow[t]{2}{*}{ No } & \multicolumn{2}{|c|}{ CXCR2 expression } & \multirow[t]{2}{*}{$P$-value } \\
\hline & & $\begin{array}{l}\text { Low (including } \\
\text { negative) }\end{array}$ & High & \\
\hline Total cases & 75 & & & \\
\hline Normal & 15 & $15(13)$ & 0 & 0 \\
\hline Glioma & 60 & $19(10)$ & 41 & \\
\hline WHO glioma grade & & & & 0.00015 \\
\hline$|-| \mid$ & 29 & $16(8)$ & 13 & \\
\hline III-IV & 31 & $3(2)$ & 28 & \\
\hline Sex & & & & 0.1521 \\
\hline Male & 36 & $13(7)$ & 23 & \\
\hline Female & 24 & $6(3)$ & 18 & \\
\hline Age (years) & & & & 0.21216 \\
\hline$\leq 45$ & 27 & $9(5)$ & 18 & \\
\hline$>45$ & 33 & $10(5)$ & 23 & \\
\hline Recurrence & & & & 0.02009 \\
\hline Recurrent & 25 & $4(2)$ & 21 & \\
\hline Primary & 35 & $15(8)$ & 20 & \\
\hline
\end{tabular}

Notes: aStatistical analyses were conducted using Fisher's exact probability. $P<0.05$ was considered as statistically significant.

Abbreviations: CXCR2, C-X-C chemokine receptor 2; WHO, World Health Organization.

Western blotting revealed that CXCR2 expression in glioma tissues was significantly higher than that in nontumor tissues (Figure 1C). This observation was significantly more apparent in high-grade gliomas (III-IV), which was increased by $782 \%$ compared with controls, while in low-grade gliomas (I-II), SASH1 expression was increased by $326 \%$ compared with controls. The differences in SASH1 expression levels between nontumor, low-grade glioma, and high-grade glioma were all statistically significant.

\section{CXCR2 expression level was significantly correlated with glioma recurrence}

The relationship between clinical characteristics and CXCR2 expression levels is shown in Table 1. Statistical analysis revealed that CXCR2 expression was not correlated with age or sex $(P>0.05)$; however, the CXCR2 expression level was significantly correlated with glioma recurrence $(P=0.02009)$. Of the 60 cases of glioma, $84.0 \%$ ( 21 in 25 cases) of recurrent glioma tissues and $57.1 \%$ (20 in 35 cases) of primary glioma tissues exhibited high CXCR2 expression.

\section{CXCR2 inhibitor SB225002 reduced cell migration}

As shown in Figure 2, we found that, after SB225002 (CXCR2 inhibitor) treatment for 0 hour, 12 hours, 24 hours, 36 hours, and 48 hours, the distance of the cell-free zone (wound zone) was $464.9 \pm 42.3 \mu \mathrm{m}, 406.7 \pm 27.4 \mu \mathrm{m}, 304.6 \pm 32.2 \mu \mathrm{m}$,
$248.8 \pm 29.3 \mu \mathrm{m}$, and $145.3 \pm 16.8 \mu \mathrm{m}$, respectively, while that of the controls was $482.5 \pm 27.4 \mu \mathrm{m}, 403.5 \pm 15.2 \mu \mathrm{m}$, $263.2 \pm 25.6 \mu \mathrm{m}, 192.9 \pm 24.8 \mu \mathrm{m}$, and $65.8 \pm 10.2 \mu \mathrm{m}$, correspondingly. It was found that after SB225002 treatment, the distance of $\mathrm{U} 251$ cell migration reduced by $3.8 \%, 11.0 \%$, $13.5 \%$, and $17.4 \%$, respectively, at 12 hours, 24 hours, 36 hours, and 48 hours. Therein, at 36 hours and 48 hours, the migration distance of SB225002-treated cells decreased significantly compared to the controls $(P<0.05)$.

\section{Discussion}

Glioma is the most common and most deadly form of primary brain tumor with a high rate of recurrence. ${ }^{22}$ Because the tumor cells invade deep into the brain itself, it is very difficult to make a complete surgical removal. Many researchers struggle to reveal the function of novel genes on invasion, metastasis, or migration of glioma cells. The ligand-receptor system on the cell surface confers an invasive or migrative phenotype in glioma cells. CXCR4, a key chemokine receptor, is reported to be implicated in glioma cell migration and invasion. ${ }^{24,25}$ Furthermore, recently, CXCR2 has been regarded as a powerful candidate gene that plays an important role in tumor migration. ${ }^{16}$

In this study, we investigated the correlation of CXCR2 expression and brain gliomas with pathological grades. The results showed that the expression of CXCR2 protein level in gliomas is significantly increased above that of nonglioma control brain tissues. Besides, the CXCR2 protein expression level was higher in high-grade gliomas than in lowgrade gliomas. This result was consistent with the reports of Korkolopoulou et al. ${ }^{17}$ We also analyzed the relationship between CXCR2 expression and patient clinicopathological characteristics, including age, sex, and recurrence, and found that the expression level of CXCR2 protein was significantly higher in recurrent patients than in primary patients. We assumed that CXCR2 may serve as a therapeutic target for glioma recurrence.

High expression levels of CXCR2 have been found in many tumor metastases and migrations. ${ }^{24,25}$ Macrophage migration inhibitory factor is often overexpressed in various malignant tumors, including melanoma and GBM, and could promote tumorigenesis. ${ }^{26,27}$ Recent work has described that migration inhibitory factor binded to CXCR2 and resulted in an integrin-dependent leukocyte arrest and mediated leukocyte chemotaxis in primary GBM cells. ${ }^{28} \mathrm{~A}$ recent study also showed that chemokine IL8 has been identified as a major secreted glioblastoma factor and drives proangiogenic and propermeability features in brain endothelial cells via 

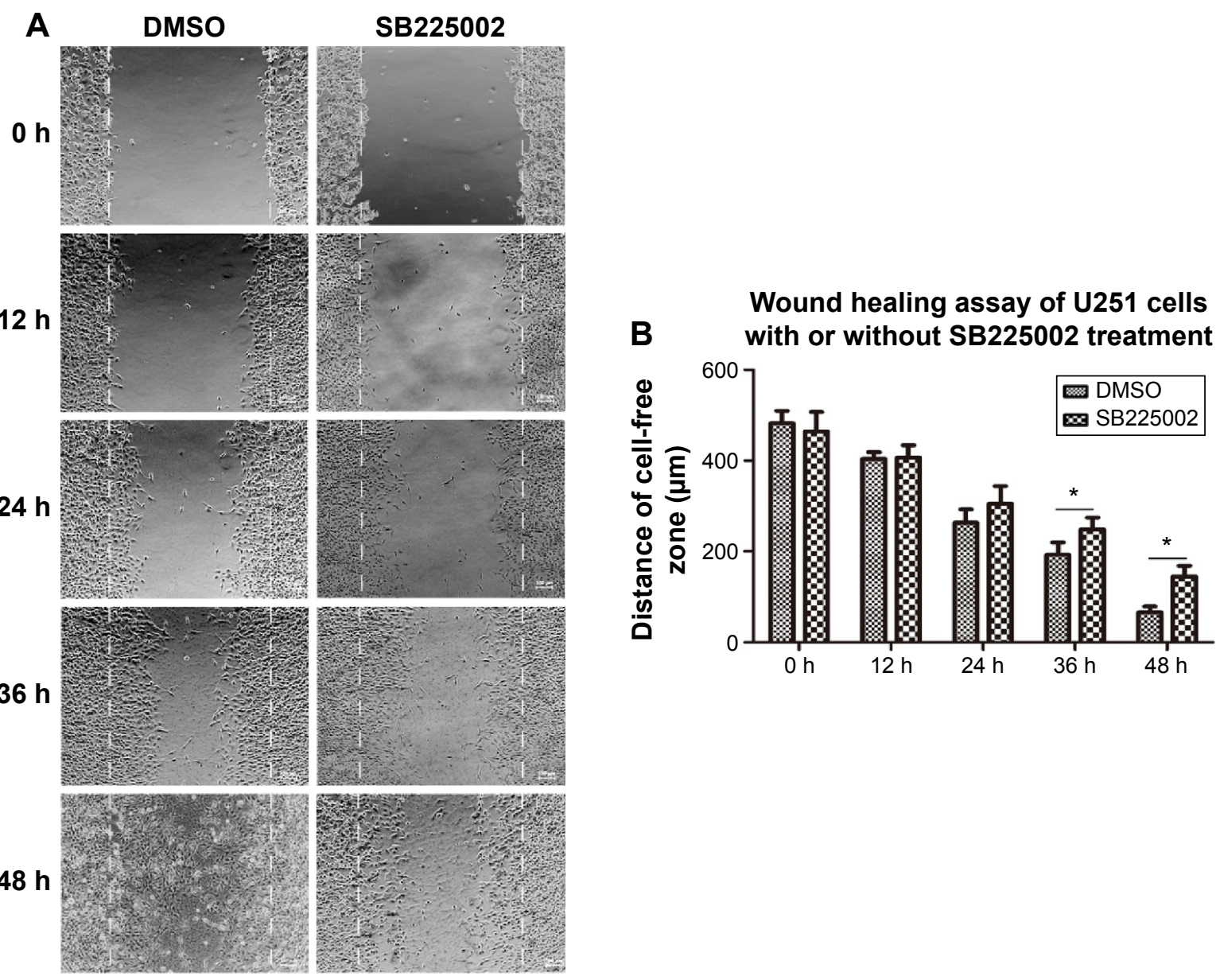

Figure 2 CXCR2 inhibitor SB225002 could reduce cell migration.

Notes: (A) Representative images for wound healing assay. U25I cells treated with DMSO or with SB225002 (400 nM) at the different time points of 0 hour, 12 hours, 24 hours, 36 hours, and 48 hours, respectively (bar $100 \mu \mathrm{m}$ ). (B) Statistical results of the wound healing assay. Images were analyzed using Image analysis software and data presented as average length of cell-free void $\pm S D(* P<0.05)$.

Abbreviations: CXCR2, C-X-C chemokine receptor 2; DMSO, dimethyl sulfoxide; SD, standard deviation; h, hour.

its receptor CXCR2. ${ }^{9}$ Therefore, we chose SB225002 to treat U251 human glioma cells to observe cells response on CXCR2 inhibition in a wound healing assay; the results suggested that $\mathrm{U} 251$ cell migration was significantly reduced. These studies underscore the importance of CXCR2 in human glioma and show that it may be a promising target for the treatment of malignant gliomas.

\section{Conclusion}

In conclusion, our results suggested that higher expressions of CXCR2 protein level in glioma were closely correlated to the degree of malignancy of gliomas and recurrence. The CXCR2 inhibitor could decrease the migration of glioma cells and may be a novel clinical target for glioma therapy.

\section{Acknowledgments}

This study was supported by grants from the National Natural Science Foundation of China (31171007), the Natural
Science Foundation of Education Department of Jiangsu Province (11KJA180004), China Postdoctoral Science Foundation (2015M571793), the Six Talent Peaks Project of Jiangsu Province (2014-WSW-027), and the Priority Academic Program Development (PAPD) of Jiangsu Higher Education Institutions.

\section{Disclosure}

The authors report no conflicts of interest in this work.

\section{References}

1. Sciume G, Santoni A, Bernardini G. Chemokines and glioma: invasion and more. J Neuroimmunol. 2010;224(1-2):8-12.

2. Sayegh ET, Kaur G, Bloch O, Parsa AT. Systematic review of protein biomarkers of invasive behavior in glioblastoma. Mol Neurobiol. 2014;49(3):1212-1244.

3. Ferguson SD. Malignant gliomas: diagnosis and treatment. Dis Mon. 2011;57(10):558-569.

4. Sherman JH, Hoes K, Marcus J, Komotar RJ, Brennan CW, Gutin PH. Neurosurgery for brain tumors: update on recent technical advances. Curr Neurol Neurosci Rep. 2011;11(3):313-319. 
5. Prasanna PG, Stone HB, Wong RS, et al. Normal tissue protection for improving radiotherapy: where are the gaps? Transl Cancer Res. 2012;1(1):35-48.

6. Tykocki T, Michalik R, Bonicki W, Nauman P. Fluorescenceguided resection of primary and recurrent malignant gliomas with 5-aminolevulinic acid. Preliminary results. Neurol Neurochir Pol. 2012;46(1):47-51.

7. Brat DJ, Bellail AC, Van Meir EG. The role of interleukin-8 and its receptors in gliomagenesis and tumoral angiogenesis. Neuro Oncol. 2005;7(2):122-133.

8. Raghuwanshi SK, Su Y, Singh V, Haynes K, Richmond A, Richardson RM. The chemokine receptors CXCR1 and CXCR2 couple to distinct $\mathrm{G}$ protein-coupled receptor kinases to mediate and regulate leukocyte functions. J Immunol. 2012;189(6):2824-2832.

9. Dwyer J, Hebda JK, Le Guelte A, et al. Glioblastoma cell-secreted interleukin-8 induces brain endothelial cell permeability via CXCR2 PLoS One. 2012;7(9):e45562.

10. Raychaudhuri B, Vogelbaum MA. IL-8 is a mediator of NF-kappaB induced invasion by gliomas. J Neurooncol. 2011;101(2):227-235.

11. Keeley EC, Mehrad B, Strieter RM. Chemokines as mediators of tumor angiogenesis and neovascularization. Exp Cell Res. 2011;317(5): 685-690.

12. Han L, Jiang B, Wu H, et al. High expression of CXCR2 is associated with tumorigenesis, progression, and prognosis of laryngeal squamous cell carcinoma. Med Oncol. 2012;29(4):2466-2472.

13. Ijichi H. Inhibition of CXCLs/CXCR2 axis in the tumor microenvironment might be a potent therapeutics for pancreatic cancer. Oncoimmunology. 2012;1(4):569-571.

14. Shang K, Bai YP, Wang C, et al. Crucial involvement of tumorassociated neutrophils in the regulation of chronic colitis-associated carcinogenesis in mice. PLoS One. 2012;7(12):e51848.

15. Lo MC, Yip TC, Ngan KC, et al. Role of MIF/CXCL8/CXCR2 signaling in the growth of nasopharyngeal carcinoma tumor spheres. Cancer Lett. 2013;335(1):81-92.

16. Saintigny $\mathrm{P}$, Massarelli E, Lin $\mathrm{S}$, et al. CXCR2 expression in tumor cells is a poor prognostic factor and promotes invasion and metastasis in lung adenocarcinoma. Cancer Res. 2013;73(2):571-582.

17. Korkolopoulou P, Levidou G, El-Habr EA, et al. Expression of interleukin-8 receptor CXCR2 and suppressor of cytokine signaling-3 in astrocytic tumors. Mol Med. 2012;18:379-388.
18. Du M, Qiu Q, Gruslin A, et al. SB225002 promotes mitotic catastrophe in chemo-sensitive and -resistant ovarian cancer cells independent of p53 status in vitro. PLoS One. 2013;8(1):e54572.

19. Sharma B, Nawandar DM, Nannuru KC, Varney ML, Singh RK. Targeting CXCR2 enhances chemotherapeutic response, inhibits mammary tumor growth, angiogenesis, and lung metastasis. Mol Cancer Ther. 2013;12(5):799-808.

20. Infanger DW, Cho Y, Lopez BS, et al. Glioblastoma stem cells are regulated by interleukin- 8 signaling in a tumoral perivascular niche. Cancer Res. 2013;73(23):7079-7089.

21. Zhou J, Yi L, Ouyang Q, Xu L, Cui H, Xu M. Neurotensin signaling regulates stem-like traits of glioblastoma stem cells through activation of IL-8/CXCR1/STAT3 pathway. Cell Signal. 2014;26(12):2896-2902.

22. Woodworth GF, Dunn GP, Nance EA, Hanes J, Brem H. Emerging insights into barriers to effective brain tumor therapeutics. Front Oncol. 2014;4:126.

23. Hulkower KI, Herber RL. Cell migration and invasion assays as tools for drug discovery. Pharmaceutics. 2011;3(1):107-124.

24. Zagzag D, Lukyanov Y, Lan L, et al. Hypoxia-inducible factor 1 and VEGF upregulate CXCR4 in glioblastoma: implications for angiogenesis and glioma cell invasion. Lab Invest. 2006;86(12):1221-1232.

25. Esencay M, Newcomb EW, Zagzag D. HGF upregulates CXCR4 expression in gliomas via NF-kappaB: implications for glioma cell migration. J Nneurooncol. 2010;99(1):33-40.

26. Shimizu T, Abe R, Nakamura H, Ohkawara A, Suzuki M, Nishihira J. High expression of macrophage migration inhibitory factor in human melanoma cells and its role in tumor cell growth and angiogenesis. Biochem Biophys Res Commun. 1999;264(3):751-758.

27. Bacher M, Schrader J, Thompson N, et al. Up-regulation of macrophage migration inhibitory factor gene and protein expression in glial tumor cells during hypoxic and hypoglycemic stress indicates a critical role for angiogenesis in glioblastoma multiforme. Am J Pathol. 2003;162(1):11-17.

28. Baron N, Deuster O, Noelker C, et al. Role of macrophage migration inhibitory factor in primary glioblastoma multiforme cells. J Neurosci Res. 2011;89(5):711-717.
OncoTargets and Therapy

\section{Publish your work in this journal}

OncoTargets and Therapy is an international, peer-reviewed, open access journal focusing on the pathological basis of all cancers, potential targets for therapy and treatment protocols employed to improve the management of cancer patients. The journal also focuses on the impact of management programs and new therapeutic agents and protocols on

\section{Dovepress}

patient perspectives such as quality of life, adherence and satisfaction. The manuscript management system is completely online and includes a very quick and fair peer-review system, which is all easy to use. Visit http://www.dovepress.com/testimonials.php to read real quotes from published authors. 